EPJ Web of Conferences 16, 04003 (2011)

DOI: $10.1051 /$ epjconf/20111604003

(C) Owned by the authors, published by EDP Sciences, 2011

\title{
Thermal tides on a hot Jupiter
}

\author{
P.-G. $\mathrm{Gu}^{1, a}$ and H.-F. Hsieh ${ }^{2}$ \\ ${ }^{1}$ Institute of Astronomy and Astrophysics, Academia Sinica, Taipei 10617, Taiwan \\ ${ }^{2}$ Institute of Astrophysics, National Taiwan University, Taipei 10617, Taiwan
}

\begin{abstract}
Following the linear analysis laid out by Gu \& Ogilvie 2009 (hereafter GO09), we investigate the dynamical response of a non-synchronized hot Jupiter to stellar irradiation. Besides the internal and Rossby waves considered by GO09, we study the Kelvin waves excited by the diurnal Fourier harmonic of the prograde stellar irradiation. We also present a 2-dimensional plot of internal waves excited by the semidiurnal component of the stellar irradiation and postulate that thermal bulges may arise in a hot Jupiter. Whether our postulation is valid and is consistent with the recent results from Arras \& Socrates (2009b) requires further investigation.
\end{abstract}

\section{INTRODUCTION}

One of the important dynamics for the large-scale flows in the planetary atmosphere is the angular momentum transport by waves (e.g., Liu \& Schneider 2009). The atmosphere of a non-synchronized hot Jupiter experiences the periodic thermal forcing as a result of alternating day-night changes. The forcing is expected to be strong since the stellar irradiation received by a hot Juptiers is remarkably intense. When the planet spins faster than its orbit, the thermal forcing is retrograde as seen by the planet. GO09 has shown that inertia gravity waves or Rossby gravity waves, depending on the asynchrony, are excited at the top of the atmosphere. These waves propagate downward and transport angular momentum upward due to radiative damping. Here we extend the work to the case that the planet spins slower than its orbit. The prograde forcing is expected to excite the Kelvin waves. The torque are calculated to compare to the previous results in Sect. 2. Another outstanding issue about these thermally driven waves is that whether these waves can generate net "thermal" bulges to drive a hot Jupiter away from synchronous rotation against gravitational tides (Arras \& Socrates 2009a) like what have been modelled for the spin of Venus (Gold \& Soter 1969). We shall discuss the existence of thermal bulges in Sect. 3 based on our preliminary calculation restricted to the radiative layer of a non-rotating hot Jupiter.

\section{KELVIN WAVES}

Figure 1 shows the latitudinal profiles of the predominant mode driven by the diurnal component $(m=1)$ of the prograde thermal forcing of periods equal to 7 and 100 days. The profiles are described by the Hough function $H_{v, 1, n^{\prime}}$ that are symmetric relative to the equation $\left(\theta=90^{\circ}\right)$ and peak at the equator, a feature of the Kelvin waves. In contrast to the Rossby waves travelling westward driven by retrograde forcing (GO09), the Kelvin waves propagate eastward. Owing to the Coriolis effect, the Kelvin waves of a longer period (100 days) are more confined to the equatorial region than those of a shorter period (7 days).

ae-mail: gu@asiaa.sinica.edu.tw

This is an Open Access article distributed under the terms of the Creative Commons Attribution-Noncommercial License 3.0, which permits unrestricted use, distribution, and reproduction in any noncommercial medium, provided the original work is properly cited. 


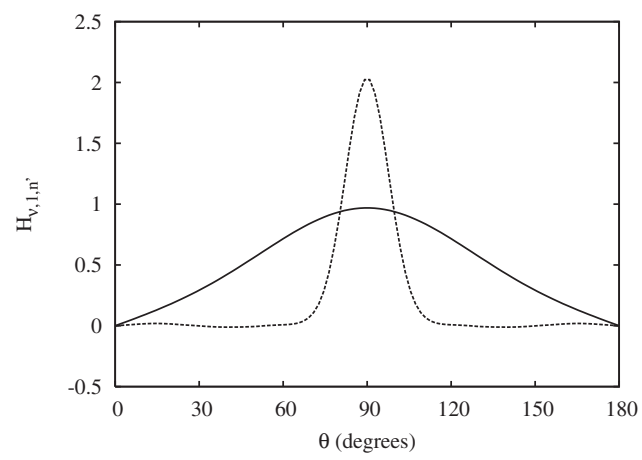

Figure 1. Latitudinal profiles of Kelvin waves driven by the diurnal thermal forcing of period 7 (solid line) and 100 days (dotted line).

Table 1. Thermal-tide torques in units of $10^{32}$ dyne $\mathrm{cm}$ generated by various waves. i-g, $\mathrm{R}$, and $\mathrm{K}$ denote inertia gravity waves, Rossby gravity waves, and Kelvin waves, respectively. A positive (negative) thermal forcing period $2 \pi / \omega$ corresponds to prograde (retrograde) forcing.

\begin{tabular}{lllll}
\hline $2 \pi / \omega$ & $-7 \mathrm{~d}$ & $-100 \mathrm{~d}$ & $7 \mathrm{~d}$ & $100 \mathrm{~d}$ \\
\hline waves $(m=1)$ & $\mathrm{i}-\mathrm{g}$ & $\mathrm{R}$ & $\mathrm{K}$ & $\mathrm{K}$ \\
thermal tidal torque & 0.001 & 14.1 & -3.5 & -28.6 \\
\hline
\end{tabular}

The radiative damping leads to the internal angular momentum transfer in the radiative layer of the hot Jupiter. Table 1 shows the torques generated by the Kelvin waves in comparison to those have been computed by GO09 for the waves excited by the retrograde thermal forcing (i.e., the inertia gravity waves for 7 days and the Rossby waves for 100 days). In contrast to retrograde waves, the downwardtravelling Kelvin waves propagate eastward and therefore transport angular momentum downward due to radiative damping as indicated by the minus signs of the torque. Unlike the inertia gravity waves, the Kelvin and Rossby waves are less confined to the equatorial region in the simulated cases and therefore are more strongly excited by the large scale thermal forcing $\propto \sin \theta$. Hence the torques arising from the damping of the Kelvin and Rossby waves can be as large as those due to the typical gravitational tides $\sim 10^{32}$ dyne $\mathrm{cm}$.

\section{POSSIBLE THERMAL BULGES?}

When the Brunt-Väisälä frequency $\gg$ the tidal forcing frequency, the waves almost attain vertically hydrostatic equilibrium and produce almost zero net thermal bulges. Nevertheless, the waves of longer wavelengths are less damped by radiative loss and thus can propagate down to the turning point near the bottom of the radiative layer where the hydrostatic balance breaks down. This suggests that the wave dynamics near the turning point may contribute to net thermal bulges. Motivated by this conjecture, we consider a plane-parallel radiative layer and ignore the Coriolis force for simplicity. We study the thermal tides arising from the semi-diurnal component of the thermal forcing. Figure 2 shows the 2$\mathrm{D}$ profile of the pressure perturbation $p^{\prime}$ associated with the internal waves excited by the retrograde forcing of period 6.3 days.

The thermal forcing (i.e. the star) is placed at $x=0$ in the figure. The wave is being thermally forced in the region $\tau<0.3$ as illustrated by the vertical patterns of $p^{\prime}$. It then starts to propagate down as an internal wave below $\tau \approx 0.3$. Note that all patterns in the figure should move to the left (i.e. westward) with the star as seen in the planet's frame. As the waves propagate down and become less damped, the wave front is tilted westward. It is because the phase velocity of internal waves is normal to its 
Research, Science and Technology of Brown Dwarfs and Exoplanets

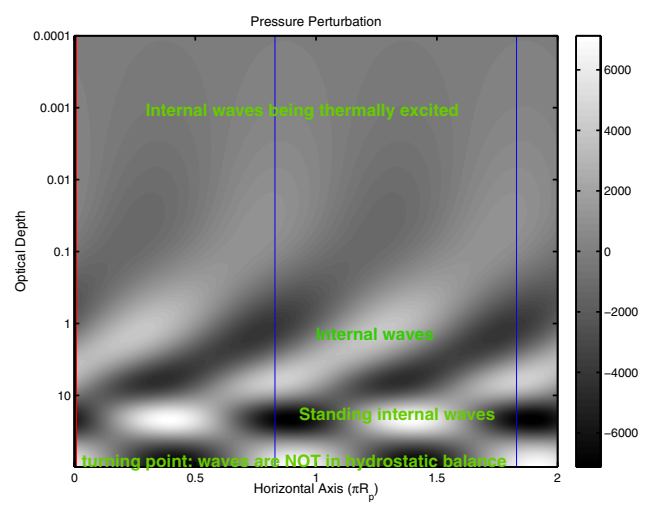

Figure 2. Profile of $p^{\prime}$ (in c.g.s. units) driven by the semi-diurnal thermal forcing of period 6.3 days.

group velocity in the absence of radiative damping. As a result, the phase velocity of internal waves propagating downward and westward should point to the up-westward direction. Since the waves of period 6.3 days can reach the turning point and reflect back, the interference between downward and upward travelling waves leads to the standing waves as illustrated by the tile-like structure of alternating $p^{\prime}$ in the region of $\tau>10$.

The perturbed column density $\Sigma^{\prime}$ is obtained by integrating density perturbation vertically throughout the radiative layer. The maximum values of $\Sigma^{\prime}$, which correspond to the locations of net thermal bulges, are denoted by the blue lines. The blue lines do NOT coincide with the maximum values of $p^{\prime}$ near the turning point, consistent with the notion that the waves are not in hydrostatic balance there. In this case, the thermal bulges (i.e. blue lines) move ahead of the stellar heating (i.e. $x=0$ ). This phase lead is due to the direction of the phase velocity. Although only the case for 6.3 days is shown, it can be expected that the phase difference varies with the vertical wavelength of waves and hence varies with the forcing period.

The result shown in Fig. 2 is obtained by imposing zero Lagrangian pressure perturbation at the bottom of the radiative layer, rather than solving the linear equations down to the planet's center. However, there might be an isostatic compensation deeper down. A calculation extended down to the convection zone is required to correctly estimate the net thermal bulges of the entire planet. Recently, Arras \& Socrates (2009b) have shown that the quadrupole moment of a hot Jupiter can be excited by the thermal forcing described by the entropy perturbation. Their eigenmode study in terms of the overlap integral shows that the primary contribution to the quadrupole moment comes from the heating layer. Unlike their approach using the entropy perturbation, we adopt the diffusive heating whose phase varies with depth. It remains to be investigated whether our argument about the contribution of the net bulges from perturbations near the turning point is valid and shares some common ground with the concept of the overlap integral.

We thank G. I. Ogilvie for useful discussions. This work is supported by the NSC grant in Taiwan via NSC 972112-M-001-017.

\section{References}

[1] Arras, P., \& Socrates, A., arXiv:0901.0735, (2009a)

[2] Arras, P., \& Socrates, A., submitted to ApJ, (2009b)

[3] Gold, T. \& Soter, S., Icarus, 11, (1969), 356

[4] Gu, P.-G., \& Ogilvie, G. I., MNRAS, 395, (2009), 422

[5] Liu, J., \& Schneider, T., submitted, (2009) 